\title{
Plasma metabolite changes in dairy cows during parturition identified using untargeted metabolomics
}

\author{
Z. Z. Luo, ${ }^{1 *}$ L. H. Shen, ${ }^{1 *}$ J. Jiang, ${ }^{1}$ Y. X. Huang, ${ }^{1}$ L. P. Bai, ${ }^{1}$ S. M. Yu, ${ }^{1}$ X. P. Yao, ${ }^{1}$ Z. H. Ren, ${ }^{1}$ Y. X. Yang, ${ }^{2}$ \\ and S. Z. Cao't \\ ${ }^{1}$ Department of Clinical Veterinary Medicine, College of Veterinary Medicine, Sichuan Agricultural University, Chengdu 611130, China \\ ${ }^{2}$ Institute of Animal Science and Veterinary Medicine, Anhui Academy of Agricultural Sciences, Hefei 230031, China
}

\section{ABSTRACT}

The metabolic responses of cows undergo substantial changes during the transition from late pregnancy to early lactation. However, the molecular mechanisms associated with these changes in physiological metabolism have not been clearly elucidated. The objective of this study was to investigate metabolic changes in transition cows from the perspective of plasma metabolites. Plasma samples collected from 24 multiparous dairy cows on approximately d 21 prepartum and immediately postpartum were analyzed using ultrahigh-performance liquid chromatography/time-of-flight mass spectrometry in positive and negative ion modes. In conjunction with multidimensional statistical methods (principal component analysis and orthogonal partial least squares discriminant analysis), differences in plasma metabolites were identified using the $t$-test and fold change analysis. Sixty-seven differential metabolites were identified consisting of AA, lipids, saccharides, and nucleotides. The levels of 32 plasma metabolites were significantly higher and those of 35 metabolites significantly lower after parturition than on d 21 prepartum. Pathway analysis indicated that the metabolites that increased from late pregnancy to early lactation were primarily involved in lipid metabolism and energy metabolism, whereas decreased metabolites were related to AA metabolism.

Key words: plasma metabolite, parturition, ultrahigh-performance liquid chromatography/time-of-flight mass spectrometry, metabolic response

\section{INTRODUCTION}

The transition period represents an important period in the production cycle of dairy cows, and generally

Received August 25, 2018.

Accepted January 10, 2019.

*These authors contributed equally to this work.

†Corresponding author: suizhongcao@126.com refers to the period extending from $21 \mathrm{~d}$ before to 21 d after calving (Drackley, 1999). During the transition from late pregnancy to early lactation, the physiological conditions, nutritional status, and metabolic responses of cows undergo marked changes. During late pregnancy, DMI decreases, which can probably be attributed to physical obstruction of the rumen by the fetus and an increase in reproductive hormone levels (Block et al., 2001; Grummer et al., 2004; Mauvais-Jarvis et al., 2013). However, large amounts of glucose are required for the synthesis and secretion of milk after calving. Accordingly, the energy demand during this period is higher than the intake, resulting in a negative energy balance (Esposito et al., 2014). The development of a negative energy balance will increase lipid mobilization and production of fatty acids that further exacerbate metabolic stress and inflammation response (Bell, 1995; Overton et al., 2017). Moreover, fatty acid production is the main factor inducing the dysfunctional inflammatory response during liver lipid mobilization and can markedly increase the risk of metritis, fatty liver, ketosis, and mastitis in postpartum cows (Contreras and Sordillo, 2011). Additionally, elevated levels of reactive oxygen species generated during lipid peroxidation will aggravate the postpartum oxidative stress response (Bernabucci et al., 2005; Sies et al., 2017). Presently, to augment the anti-stress and anti-inflammatory abilities of cows during calving, dietary supplementation strategies are used, including the provision of selenium, vitamin E, and n-3 PUFA, which might also reduce the risk of postpartum illness (Abuelo et al., 2015; Khatti et al., 2017; Schäfers et al., 2018). Given that the physiological mechanisms associated with transition in dairy cows are not comprehensively understood, only limited measures are available to relieve transitional stress. Researchers have attempted to solve this problem using omics approaches, particularly metabolomics, from 2 perspectives: (1) the first involves gaining a better understanding of disease pathogenesis based on metabolomics to identify blood metabolites that differ between diseased and healthy cows, thereby providing information for initiating preventive measures. This method has been 
widely used to address ketosis, metritis, placenta retention, and fatty liver problems in dairy cows (Zhang et al., 2013, 2017a,b; Imhasly et al., 2014; Sun et al., 2014; Dervishi et al., 2017a,b). (2) The second perspective involves intensive study of the changes in metabolite levels in blood at different stages during the production cycle. Previous studies have assessed the dynamic changes in certain AA, biogenic amines, acylcarnitines, phosphatidylcholines, and sphingomyelins during the transition from nonlactating to lactating stages based on targeted metabolomics $(-42,-10,3,21$, and $100 \mathrm{~d}$; Kenéz et al., 2016), and it has accordingly been demonstrated that concentrations of glycerophospholipids and sphingolipids are significantly lower at $10 \mathrm{~d}$ before and $3 \mathrm{~d}$ after calving than at earlier or later stages in the transition period. However, few studies have shown changes in global metabolites and metabolic pathways during the transitional periods.

Metabolomics was first systematically introduced by Nicholson et al. (1999) and has subsequently evolved into untargeted and targeted approaches. Targeted metabolomics is used to quantitatively detect metabolites in metabolic pathways of interest, whereas untargeted metabolomics examines metabolite differences between control and experimental groups, which also plays an important role in marker screening for disease (Patti et al., 2012; Li et al., 2017). In this study, we used an untargeted metabolomics approach to analyze plasma metabolite profiles in transition cows and identify differential metabolites at 2 crucial time points $(21 \mathrm{~d}$ before the due date and on the calving day) in the dairy cow production cycle. The identification of differential metabolites may provide novel information for elucidating physiological responses and characterizing new pathways that may be initiated under parturition stress.

\section{MATERIALS AND METHODS}

\section{Sample Collection}

Sample collection was performed in strict accordance with the guidelines of the Care and Use of Laboratory Animals of China, and all procedures were approved by the Animal Care and Use Committee of Sichuan Agricultural University. For the experimental animals, we selected 24 healthy multiparous Holstein dairy cows with similar 305-d milk yields, parity, BCS, and due date from Puzhou Dairy Farm (Sichuan, China). The cows were housed in freestall barns and had free access to fresh water. A TMR diet was provided daily at 0600,1200 , and $1900 \mathrm{~h}$. The ingredients and chemical composition of the prepartum TMR are shown in Supplemental Table S1 (https://doi.org/10.3168/jds
.2018-15601). Blood was collected via the caudal vein at $-21 \mathrm{~d}$ ( $21 \mathrm{~d}$ before the due date) before the morning feeding and on the day of calving within $6 \mathrm{~h}$ after the milking colostrum. Plasma was collected using EDTA as an anticoagulant, then centrifuged at $1,500 \times g$ for 10 min at room temperature, and subsequently stored at $-80^{\circ} \mathrm{C}$. Samples were divided into 2 groups according to sampling time: $21 \mathrm{~d}$ before the due date $(\mathbf{P}-21$ d) and after calving $(\mathbf{P}-\mathbf{0} \mathbf{d})$.

\section{Sample Pretreatment}

Forty-eight plasma samples were slowly thawed at $4^{\circ} \mathrm{C}$, and from each sample a $100-\mu \mathrm{L}$ aliquot was taken and added to $400 \mu \mathrm{L}$ of a pre-cooled methanol/acetonitrile solution $(1: 1, \mathrm{vol} / \mathrm{vol})$. Samples were then vortex mixed and maintained at $-20^{\circ} \mathrm{C}$ for $60 \mathrm{~min}$, followed by centrifugation at $14,000 \times g$ and $4^{\circ} \mathrm{C}$ for $20 \mathrm{~min}$. The supernatant fraction was collected and dried. The dried metabolites were dissolved by adding $100 \mu \mathrm{L}$ of aqueous acetonitrile (acetonitrile: water $=1: 1, \mathrm{vol} / \mathrm{vol}$ ), vortex mixed, and centrifuged at $14,000 \times g$ and $4^{\circ} \mathrm{C}$ for $15 \mathrm{~min}$. The resulting supernatant was collected and analyzed. The 2 sets of treated samples were mixed in equal amounts for the preparation of quality control (QC) samples, and 6 replicates were set up to evaluate system stability over the entire experiment before testing. After the completion of sample pretreatment, the samples were sent to Shanghai Applied Protein Technology Co., Ltd. (Shanghai, China) for liquid chromatography-tandem mass spectrometry (MS/ MS) analysis.

\section{Ultra-High-Performance Liquid Chromatography Time-of-Flight/MS Analysis}

Samples after pretreatment were separated using an ultra-high-performance liquid chromatography (UHPLC) system (1290 Infinity II, Agilent Technologies, Santa Clara, CA) incorporating an HILIC column (2.1 $\mathrm{mm} \times 100 \mathrm{~mm}, 1.7 \mu \mathrm{m}$; Waters, Milford, MA). The column temperature was $25^{\circ} \mathrm{C}$, and we used a flow rate of $0.3 \mathrm{~mL} / \mathrm{min}$. The mobile phase consisted of $\mathrm{A}$ (water $+25 \mathrm{mmol} / \mathrm{L}$ of ammonium acetate $+25 \mathrm{mmol} / \mathrm{L}$ of ammonia) and B (acetonitrile). The gradient elution procedure was as follows: $0-1 \mathrm{~min}, 95 \% \mathrm{~B} ; 1-14 \mathrm{~min}$, $95 \%$ to $65 \% \mathrm{~B} ; 14-16 \mathrm{~min}, 65 \%$ to $40 \% \mathrm{~B} ; 16-18 \mathrm{~min}$, $40 \% \mathrm{~B} ; 18-18.1 \mathrm{~min}, 40 \%$ to $95 \% \mathrm{~B}$; and $18.1-23 \mathrm{~min}$, $95 \% \mathrm{~B}$. The autosampler was maintained at $4^{\circ} \mathrm{C}$ and the injection volume was $2 \mu \mathrm{L}$. The $\mathrm{QC}$ samples were inserted into the samples to monitor system stability and data quality.

The samples were analyzed using a triple time-of-flight (TOF) $5600^{+}$system (AB/SCIEX, Framingham, MA) 
equipped with an electrospray ionization source used in positive and negative ion modes. The mass spectrometry detection variables were as follows: gas 1, 0.4137 MPa; gas 2, 0.4137 MPa; curtain gas, $0.20685 \mathrm{MPa}$; ion source temperature, $600^{\circ} \mathrm{C}$; ionization voltage, $\pm 5,500$ $\mathrm{V}$; TOF-MS scan range, $60-1,000 \mathrm{~m} / z$; precursor ion scan range, 25-1,000 $\mathrm{m} / z$; scan accumulation time, 0.2 $\mathrm{s} /$ spectrum; precursor ion scan accumulation time, 0.05 $\mathrm{s} /$ spectrum; declustering potential, $60 \mathrm{~V}$; and collision energy, $35 \pm 15 \mathrm{eV}$. Tandem mass spectrometry data were acquired in the information-dependent acquisition mode and high sensitivity modes were used.

\section{Data Processing and Statistical Analyses}

The raw data were converted into the mzXML format using ProteoWizard (Chambers et al., 2012), and then peak alignment, retention time correction, and peak area extraction were performed using the $\mathrm{R}$ package XCMS (Jia et al., 2018). For the data extracted using XCMS, ion peak data for which $>50 \%$ of the data were missing within a group were deleted. After the data had been pre-processed by pareto-scaling, pattern recognition was performed using SIMCA-P software (version 14.1, Umetrics, Umea, Sweden), consisting of unsupervised principal component analysis (PCA) and supervised orthogonal partial least squares discriminant analysis (OPLS-DA). Principal component analysis was used to determine intra-group aggregation and inter-group separation tendencies, whereas OPLSDA was performed to further determine inter-group differences. The OPLS-DA models were validated based on interpretation of variation in $\mathrm{Y}\left(\mathbf{R}^{2} \mathbf{Y}\right)$ and forecast ability based on the model $\left(\mathbf{Q}^{2}\right)$ in cross-validation and permutation tests by applying 200 iterations. When 1 $\geq \mathrm{R}^{2} \mathrm{Y}$ and $\mathrm{Q}^{2} \geq 0.4$, the models were determined to be stable and reliable (Westerhuis et al., 2008). In addition, a $\mathrm{Q}^{2}$ intercept $<0.05$ from the permutation test was used to verify that there was no overfitting (Liu et al., 2015), and univariate analysis was performed, including Student's $t$-test and fold change analysis.

\section{Metabolite Identification and Pathway Analysis}

Significantly differential metabolites were screened using variable importance in projection (VIP) scores (VIP $>1$ ) obtained from the OPLS-DA model and $P$ values $(P<0.05)$. Identification of differential metabolites was carried out by searching an in-house standard MS/MS library and the online database METLIN (http://metlin.scripps.edu/) using MS/MS spectra or exact mass data (Jia et al., 2018). The in-house library contains MS/MS spectra of approximately $800 \mathrm{com}-$ pounds, which were obtained from standards. The MS/
MS spectra matching score was calculated using the dot-product algorithm and the score cutoff was set as 0.8 (Stein and Scott, 1994; Jia et al., 2018). The MS/ MS spectra that could not be matched to any of those in the in-house library were searched in online databases. Mass error was set within $25 \mathrm{ppm}$. Moreover, clustering and pathway analyses data were processed and analyzed using MetaboAnalyst 4.0 (http://www .metaboanalyst.ca).

\section{RESULTS}

\section{Metabolite Profiles of Plasma Samples and Data Analysis}

We compared the total ion chromatograms (TIC) of 6 QC samples in positive or negative ion modes, including the retention time (RT), peak, intensity, and degree of separation. Overlap of the TIC of QC samples was good, indicating that the method used was robust, with high repeatability and stability. The sample TIC showed that the peak shape was intact and that adjacent peaks were well separated from each other, indicating that the chromatographic and mass spectrometric conditions were suitable for sample identification (Supplemental Figure S1; https://doi.org/10 .3168/jds.2018-15601).

The PCA score plot showed that the model interpretation rates for the $\mathrm{P}-0 \mathrm{~d}$ and $\mathrm{P}-21 \mathrm{~d}$ groups under the positive and negative ion mode conditions were $\mathrm{R}^{2} \mathrm{X}=$ 0.525 and 0.565 , respectively. The 2 groups of samples were well separated, and samples in the same group were well aggregated together (Figure 1A and 1B). A OPLS-DA supervised model was used to assess intergroup sample differences. In the positive ion mode of the OPLS-DA score plot, $R^{2} Y=0.986$ and $Q^{2}=0.930$, whereas in the negative ion mode, $R^{2} Y=0.982$ and $Q^{2}$ $=0.939$. Both $\mathrm{R}^{2} \mathrm{Y}$ and $\mathrm{Q}^{2}$ values were greater than 0.4 , indicating that the model was stable and reliable (Figure $2 \mathrm{~A}$ and $2 \mathrm{~B}$ ). $\mathrm{A} \mathrm{Q}^{2}$ value of approximately 1 indicated that the OPLS-DA model had good predictability. The $\mathrm{Q}^{2}$ intercept values were less than 0.05 , indicating that there was no overfitting (Figure $2 \mathrm{C}$ and 2D).

\section{Differential Metabolite Analysis}

A VIP score $>1$ and $P<0.05$ were used as criteria for differential metabolite screening. We identified a total of 67 differential metabolites, of which 37 were detected in the positive ion mode and 30 in the negative ion mode (Tables 1 and 2). Of these, the levels of 32 metabolites had increased, whereas those of 35 had decreased at P-0 d with respect to the levels at P-21 
d. A generated cluster heat map showed that similar metabolites were located in close proximity, and a dendrogram indicated that the samples of the P-0 d and P-21 d groups can be separated (Figure 3A and 3B).

We subsequently queried differential metabolites in the Kyoto Encyclopedia of Genes and Genomes pathway database and searched published articles for data relating to global metabolism (Figure 4). The global metabolic pathways identified are involved in lipid metabolism, energy metabolism, AA metabolism, and nucleotide metabolism, and 3 reaction pathways were analyzed: gluconeogenesis, urea cycle, and tricarboxylic acid (TCA) cycle. To further determine the biological significance of the differential metabolites, we performed a metabolic pathway analysis using MetaboAnalyst software. A total of 10 pathway impacts $>0.2$ with $P$ $<0.05$ were observed for the main metabolic pathway, among which 3 pathways had a pathway impact value of 1: linoleic acid (LA) metabolism; Val-Leu and Ile biosynthesis; and D-Gln and D-glutamate metabolism (Figure 5).

\section{DISCUSSION}

Several previous studies have indicated that inflammatory responses and oxidative stress are common occurrences around the time of calving in dairy cows (Contreras et al., 2010; Sharma et al., 2011; Trevisi et al., 2012). Lipid mediators derived from lipid metabolism play an important role in the inflammatory responses of cows during calving (Sordillo and Mavangira, 2014; Mavangira and Sordillo, 2018). And among these, the oxylipid derived from the n-6 PUFA enzymatic pathway is involved in the initiation of inflammation and pro-inflammation (Marion-Letellier et al.,
2015). Although we did not identify any oxylipids in the present study, we did identify several important precursors in the pathway, including LA, arachidonic acid, $\gamma$-linolenic acid, and phosphatidylcholine (PC). These lipids were upregulated postpartum, which is consistent with the findings of previous studies. Some previous studies have indicated that eicosanoids derived from arachidonic acid via the cyclooxygenase/lipoxygenase pathway, including leukotrienes, prostaglandins, and hydroxyeicosatetraenoic acids, may act as regulators of inflammation (Raphael et al., 2014; Mavangira et al., 2015; Ryman et al., 2015). As a major n-6 PUFA, LA can be used as a precursor of arachidonic acid via dehydrogenase catalysis. Moreover, it can be derived from hydroperoxyoctadecadienoic acid to promote inflammation via the 15-LOX pathway (Gulliver et al., 2012; Ryman et al., 2016). The PC metabolism has been shown to be enhanced under inflammatory conditions and increases the production of arachidonic acid and LA via the action of a phospholipase (Dennis and Norris, 2015; Leng et al., 2018). It has also been reported that LA, oleic acid, and palmitic acid levels are significantly elevated in the serum of cows with mastitis, which is consistent with the lipid changes identified in the present study (Dervishi et al., 2017a). Furthermore, we found that several lysophosphatidylcholines (LysoPC) were reduced in the PC metabolic pathway via the phospholipase A2 (PLA2) pathway. This is consistent with the observed significant decreases in LysoPC, including LysoPC14:0, LysoPC16:0, LysoPC18:0, and LysoPC18:1, when cows enter the lactation stage, as reported by Kenéz et al. (2016). Studies have also shown that, compared with 4 wk before calving, both LysoPC C20:4 and C28:0 decreased after calving, whereas cows with a retained placenta show a
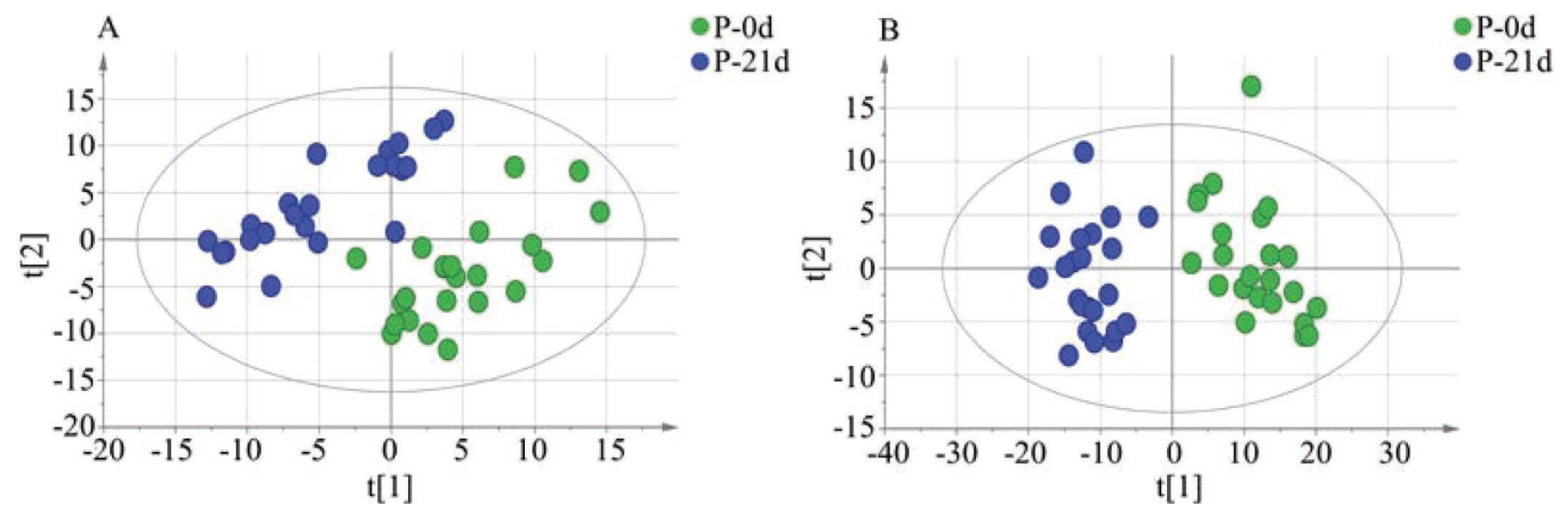

Figure 1. (A) Principal component analysis score plot for the P-0 d (sampling time after calving) and P-21 d group (sampling time $21 \mathrm{~d}$ before the due date) samples analyzed in the positive ion mode. (B) Principal component analysis score plot for the P-0 d and P-21 d group samples analyzed in the negative ion mode. $\mathrm{t}[1]=$ first principal component. $\mathrm{t}[2]=$ second principal component. 
decreasing trend at 8 wk before calving (Dervishi et al., 2017b). However, research on these types of LysoPC has indicated that pro-inflammatory cytokine vascular cell adhesion molecule- 1 promotes the production of LysoPC16:0, LysoPC18:0, and LysoPC18:1, and that this activity is inhibited by the action of oleyloxyethyl phosphorylcholine, a PLA2-selective inhibitor (Zhuge et al., 2014). The observed decreases in levels of LysoPC after parturition may thus be related to the action of PLA2-selective inhibitors, and further research is needed in this regard.

Previous studies have confirmed that the DMI of dairy cows is significantly reduced during the transition period, particularly immediately before and after parturition (Bell, 1995; Gandra et al., 2014; Cheong et al., 2016). However, when cows enter the lactation stage, energy intake is insufficient to meet the energy demand for lactation, and thus the body will increase the pro- duction of endogenous glucose to maintain glucose homeostasis (Wankhade et al., 2017). Scholars have found that blood glucose levels in cows increase significantly after parturition (Salin et al., 2017). Consistently, in the present study, we observed that glucose levels in the plasma of dairy cows also increased significantly after parturition. Moreover, we found that levels of lactic acid, which is a TCA cycle precursor, also increased significantly in the gluconeogenesis pathway. We also suspect that uridine in the nucleotide metabolic pathway is involved in insulin resistance. Uridine is a pyrimidine nucleotide synthesized from pyrimidine and ribose, and can be phosphorylated to produce uridine triphosphate that acts in concert with glucose-1-phosphate to produce uridine diphosphate glucose, which is involved in glycogen synthesis (Connolly and Duley, 1999; Roach et al., 2012). Uridine plays a role in insulin resistance and promotes gluconeogenesis, largely through an in-
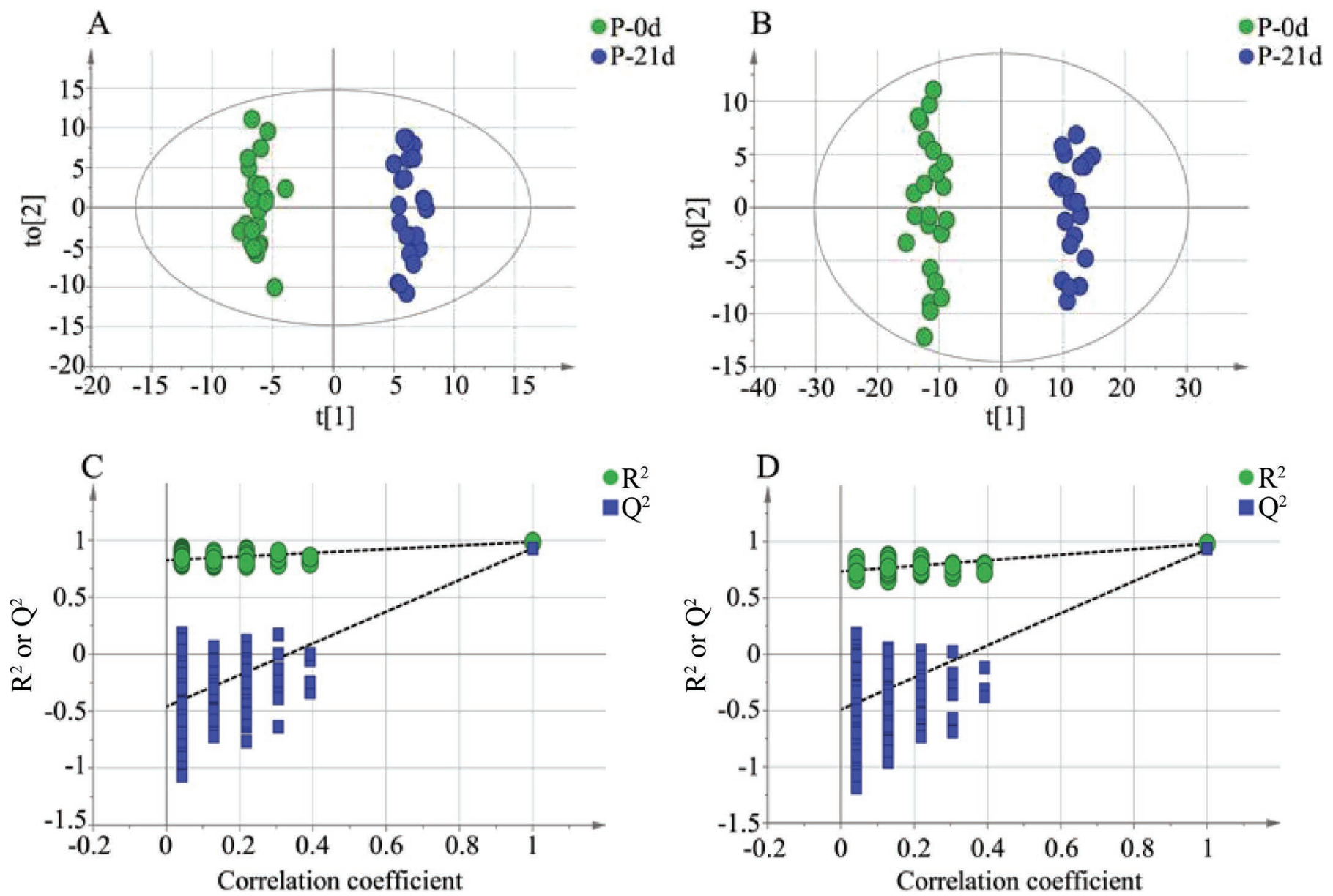

Figure 2. (A) and (C) Orthogonal partial least square discriminant analysis (OPLS-DA) of scores and permutation test plots for the P-0 d (sampling time after calving) and P-21 d (sampling time $21 \mathrm{~d}$ before the due date) group samples analyzed in the positive ion mode, respectively. (B) and (D) Orthogonal partial least square discriminant analysis of scores and permutation test plots for the P-0 d and P-21 d group samples analyzed in the negative ion mode, respectively. $\mathrm{t}[1]=$ first principal component. to $[2]=$ second orthogonal component. The intercept limit of $\mathrm{Q}^{2}$, calculated by regression line, is the plot of $\mathrm{Q}^{2}$ from permutation test in the OPLS-DA model. 
LUO ET AL.

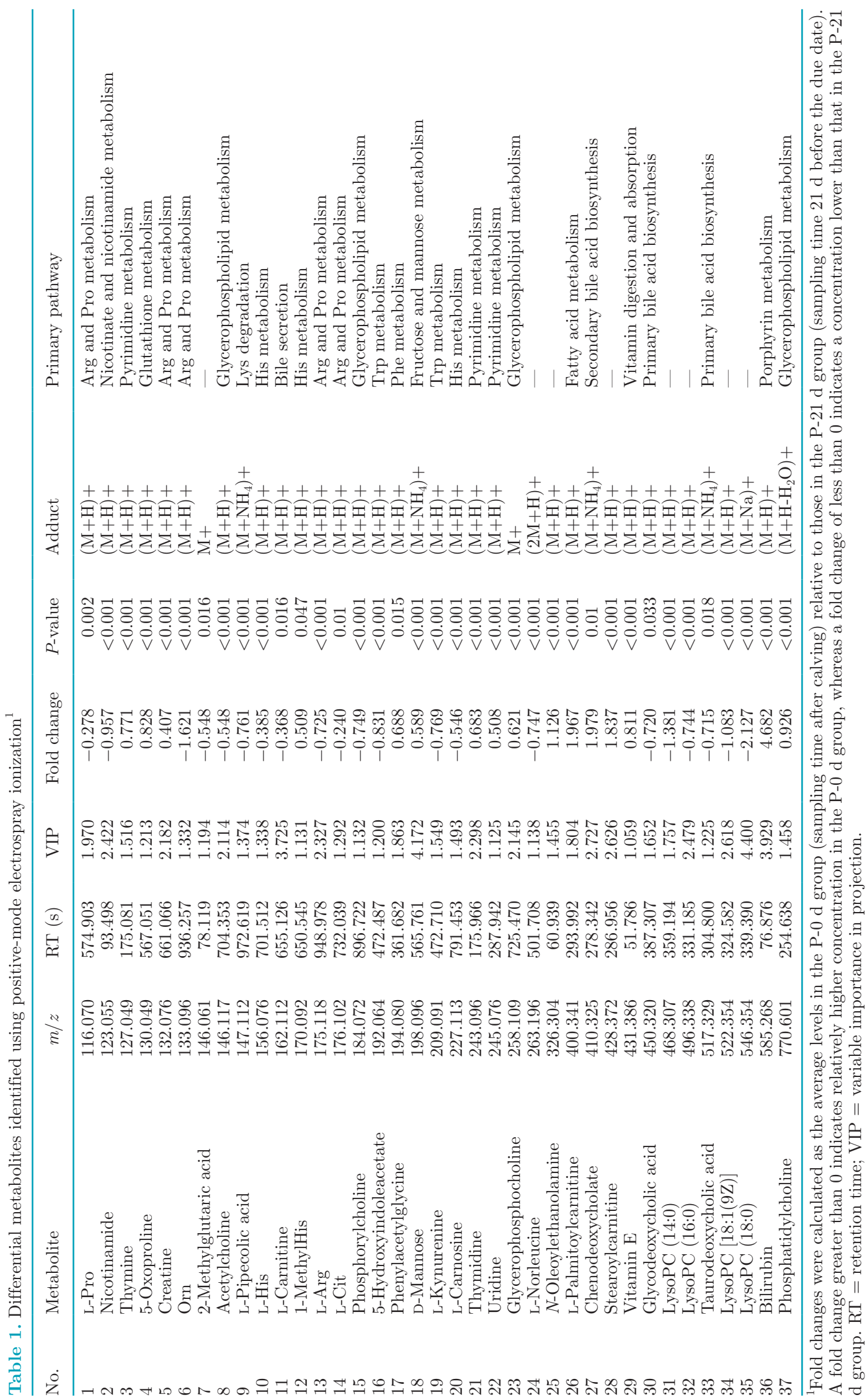




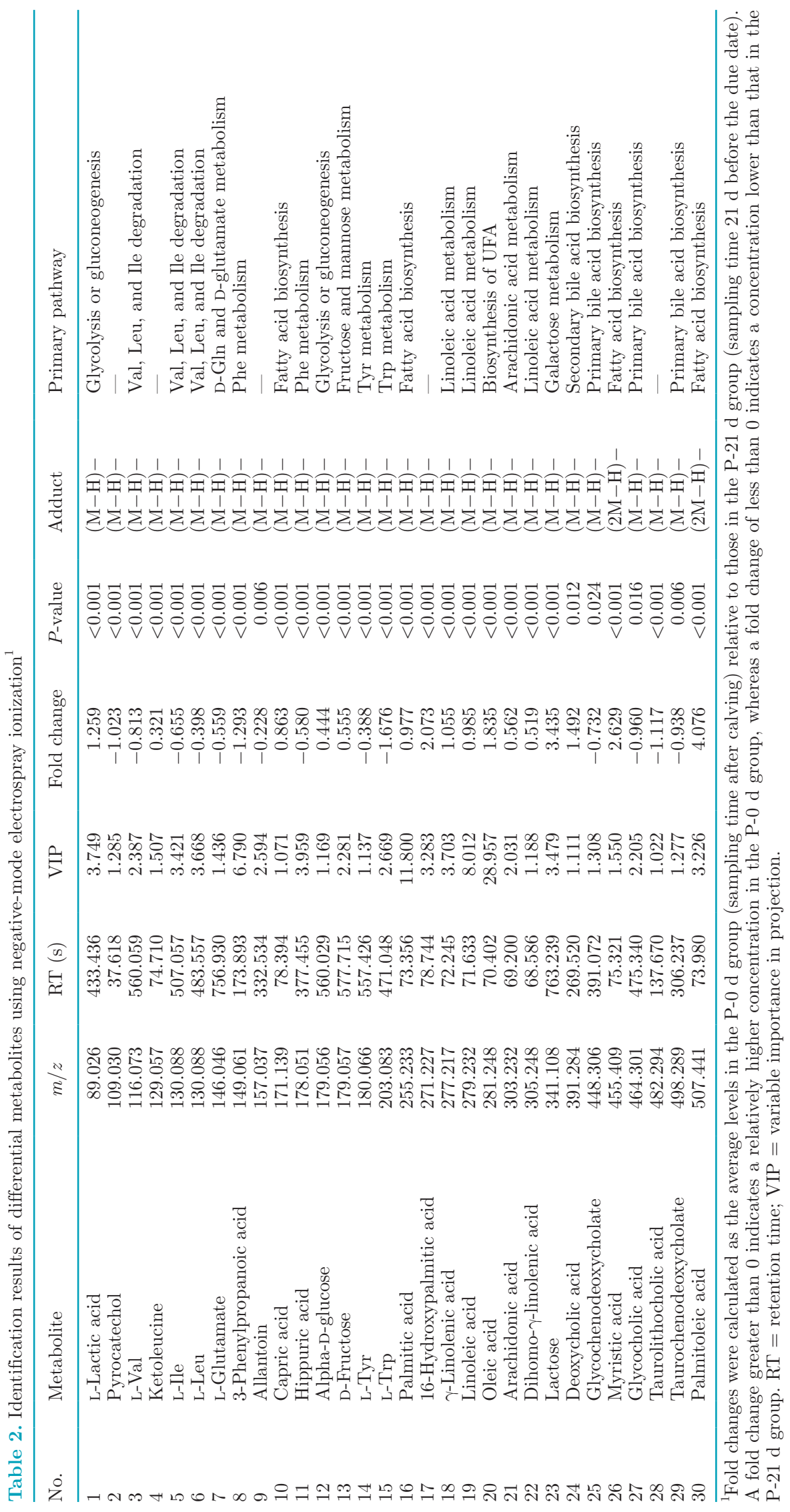



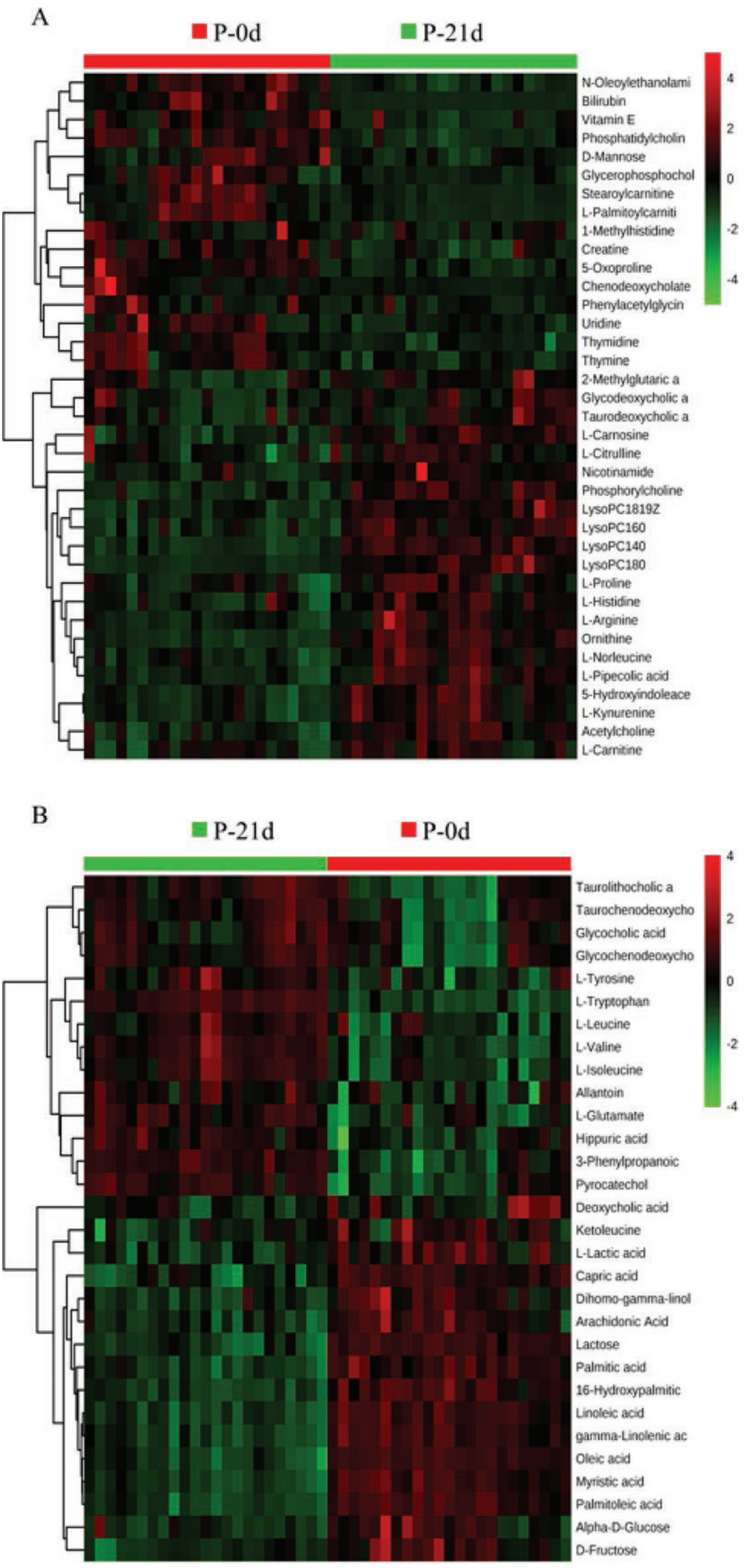

Figure 3. Heatmap of the 37 and 30 differential metabolites identified in serum samples analyzed in positive and negative ion modes, respectively. P-0 d = sampling time after calving; P-21 d = sampling time $21 \mathrm{~d}$ before the due date. 
crease in liver protein glycosylation and a reduction in protein phosphorylation in the hepatic insulin signaling pathway (Park et al., 2005; Urasaki et al., 2014). Thus, we believe that nucleotide metabolism is involved in glucose homeostasis after parturition in dairy cows.

Amino acids are important substances in the synthesis of tissue proteins and milk components. Previous studies have indicated that certain AA can affect cow performance and milk quality (Robinson et al., 2010; Lee et al., 2015; Giallongo et al., 2016). However, limited information is currently available regarding the AA metabolism of dairy cows, particularly during the transition period. Although $21 \mathrm{AA}$ were detected in a study conducted by Kenéz et al. (2016), none of these were observed to undergo significant changes during the prepartum and postpartum periods. In the pres- ent study, however, we found that certain AA in the plasma of cows, including Val, Ile, Leu, His, Trp, Glu, and Arg, underwent significant changes during parturition. Furthermore, we found that the urea cycle may be affected during parturition. Previous studies have indicated that the levels of Arg and Glu in the urea cycle are closely related to the synthesis of antioxidant and anti-inflammatory molecules (Jiao et al., 2015; Liang et al., 2018; Zhao et al., 2018). Moreover, we found that certain AA affect gluconeogenesis in dairy cows after calving by participating in the TCA cycle. Consistently, Arg, Pro, and Glu in the urea cycle have previously been shown to participate in the TCA cycle by affecting the formation of fumarate (Sugiyama et al., 2014; Yoshimi et al., 2016). We found that other AA involved in the TCA cycle, including Leu and Trp,

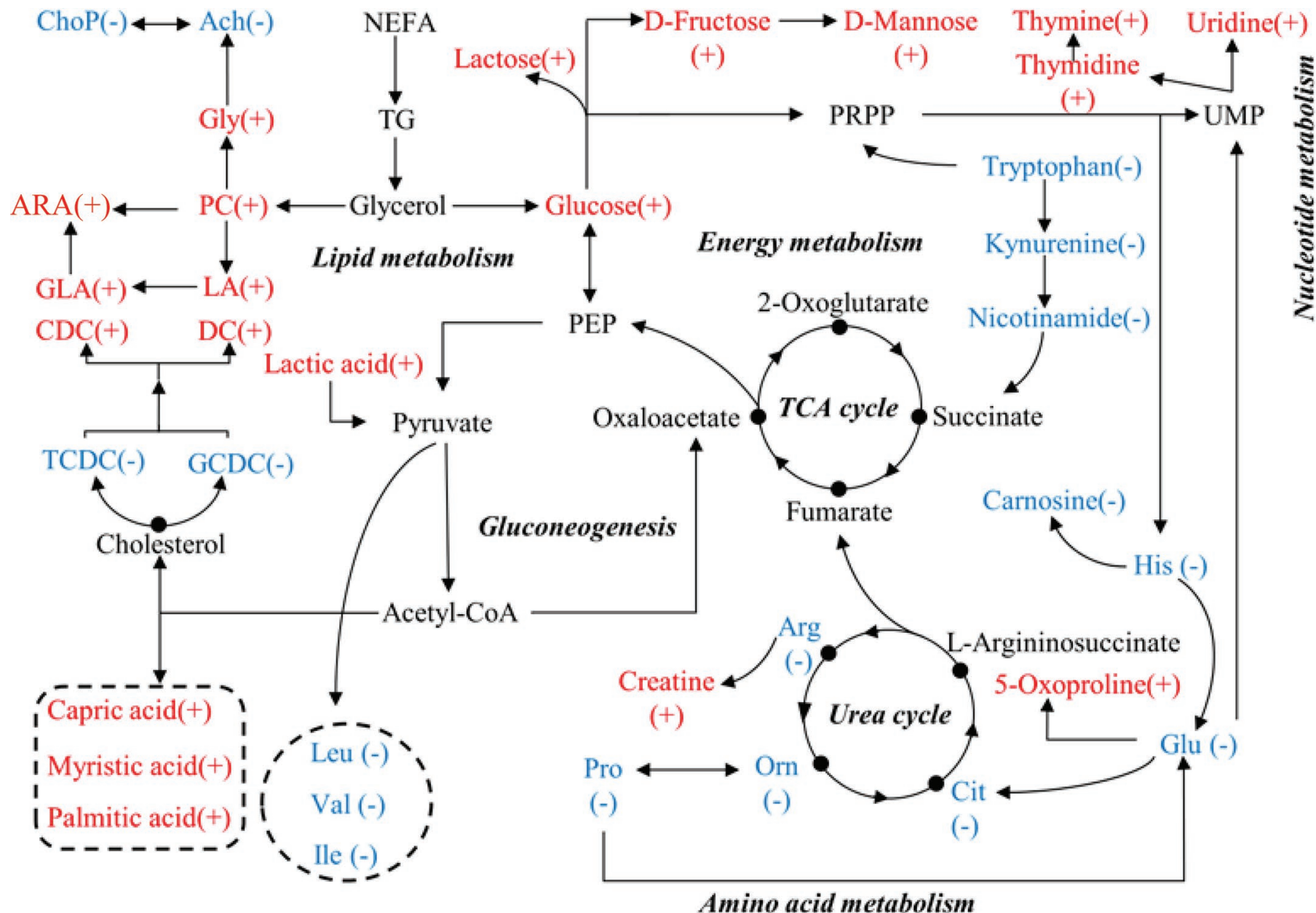

Figure 4. Metabolic pathway for differential metabolites, mainly for lipid metabolism, energy metabolism, amino metabolism, and nucleotide metabolism. (+) indicates higher concentrations during calving; (-) indicates lower concentrations than those observed at d 21 prepartum. $\mathrm{ARA}=$ arachidonic acid; GLA = gamma linolenic acid; LA = linoleic acid; NEFA = nonesterified fatty acids; PC = phosphatidylcholine; Gly $=$ glycerophosphocholine; $\mathrm{Ach}=$ acetylcholine; $\mathrm{ChoP}=$ phosphorylcholine; $\mathrm{TG}=$ triglyceride; $\mathrm{PEP}=$ phosphoenolpyruvate; $\mathrm{PRPP}=5$-phosphoribosyl 1-pyrophosphate; UMP = uridine monophosphate; TCDC = taurochenodeoxycholate; GCDC = glycochenodeoxycholate; CDC $=$ chenodeoxycholate; DC = deoxycholic acid; TCA = tricarboxylic acid. 


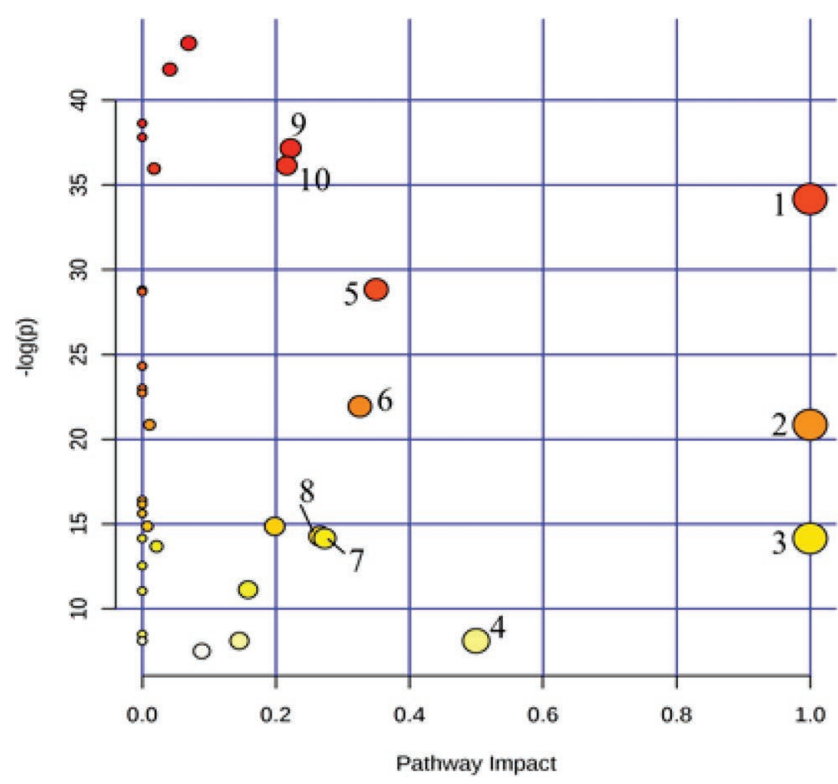

1 Linoleic acid metabolism

2 Val, Leu, and Ile metabolism

3 D-Gln and D-glutamate metabolism

4 Phe, Tyr, and Trp biosynthesis

5 Arg and Pro metabolism

6 Arachidonic acid metabolism

7 Ala, Asp, and Gln metabolism

8 His metabolism

9 Try metabolism

10 Glycerophospholipid metabolism

Figure 5. Metabolic pathway analysis using MetaboAnalyst 4.0 (http://www.metaboanalyst.ca). x-axis, pathway impact; y-axis, - log ( $P$ ). Circles represent metabolic pathways. Darker circles indicate more significant changes in the metabolites in the corresponding pathway, whereas the size of the circle corresponds to the pathway impact score.

also undergo similar changes after calving, whereas previous studies have found that levels of the AA His, Leu, Lys, Pro, and Trp are significantly increased in the serum of cows with ketosis (Sun et al., 2014; Zhang et al., 2017b), thereby indicating that the urea cycle participates in the gluconeogenesis of cows in response to a negative energy balance. However, the specific mechanisms underlying the AA metabolism involved in energy metabolism during calving need further investigation.

\section{CONCLUSIONS}

In this study, a metabolomics approach based on UHPLC-TOF/MS analysis was used to study metabolic changes in transition dairy cows. We accordingly identified 67 metabolites that showed differential expression between d 21 before the due date and after calving. Furthermore, we found that lipid, glucose, and nucleotide metabolism increased after calving, whereas AA metabolism decreased. Further studies should use targeted metabolomics to verify metabolite levels and to analyze related regulatory enzymes in metabolic pathways of interest.

\section{ACKNOWLEDGMENTS}

This project was supported by the Double Subject Construction Plan of Sichuan Agricultural University (no. 03571537). We thank Shanghai Applied Protein
Technology Co. Ltd. (Shanghai, China) for UHPLCTOF/MS analysis.

\section{REFERENCES}

Abuelo, A., J. Hernandez, J. L. Benedito, and C. Castillo. 2015. The importance of the oxidative status of dairy cattle in the periparturient period: Revisiting antioxidant supplementation. J. Anim. Physiol. Anim. Nutr. (Berl.) 99:1003-1016.

Bell, A. W. 1995. Regulation of organic nutrient metabolism during transition from late pregnancy to early lactation. J. Anim. Sci. 73:2804-2819

Bernabucci, U., B. Ronchi, N. Lacetera, and A. Nardone. 2005. Influence of body condition score on relationships between metabolic status and oxidative stress in periparturient dairy cows. J. Dairy Sci. 88:2017-2026.

Block, S. S., W. R. Butler, R. A. Ehrhardt, A. W. Bell, M. E. Van Amburgh, and Y. R. Boisclair. 2001. Decreased concentration of plasma leptin in periparturient dairy cows is caused by negative energy balance. J. Endocrinol. 171:339-348.

Chambers, M. C., B. Maclean, R. Burke, D. Amodei, D. L. Ruderman, S. Neumann, L. Gatto, B. Fischer, B. Pratt, J. Egertson, K. Hoff, D. Kessner, N. Tasman, N. Shulman, B. Frewen, T. A. Baker, M.-Y. Brusniak, C. Paulse, D. Creasy, L. Flashner, K. Kani, C. Moulding, S. L. Seymour, L. M. Nuwaysir, B. Lefebvre, F. Kuhlmann, J. Roark, P. Rainer, S. Detlev, T. Hemenway, A. Huhmer, J. Langridge, B. Connolly, T. Chadick, K. Holly, J. Eckels, E. W. Deutsch, R. L. Moritz, J. E. Katz, D. B. Agus, M. MacCoss, D. L. Tabb, and P. Mallick. 2012. A cross-platform toolkit for mass spectrometry and proteomics. Nat. Biotechnol. 30:918.

Cheong, S. H., O. G. Sa Filho, V. A. Absalon-Medina, S. H. Pelton, W. R. Butler, and R. O. Gilbert. 2016. Metabolic and endocrine differences between dairy cows that do or do not ovulate first postpartum dominant follicles. Biol. Reprod. 94:18.

Connolly, G. P., and J. A. Duley. 1999. Uridine and its nucleotides: Biological actions, therapeutic potentials. Trends Pharmacol. Sci. $20: 218-225$.

Contreras, G. A., N. J. O'Boyle, T. H. Herdt, and L. M. Sordillo. 2010. Lipomobilization in periparturient dairy cows influences the com- 
position of plasma nonesterified fatty acids and leukocyte phospholipid fatty acids. J. Dairy Sci. 93:2508-2516.

Contreras, G. A., and L. M. Sordillo. 2011. Lipid mobilization and inflammatory responses during the transition period of dairy cows. Comp. Immunol. Microbiol. Infect. Dis. 34:281-289.

Dennis, E. A., and P. C. Norris. 2015. Eicosanoid storm in infection and inflammation. Nat. Rev. Immunol. 15:511-523.

Dervishi, E., G. Zhang, S. M. Dunn, R. Mandal, D. S. Wishart, and B. N. Ametaj. 2017a. GC-MS metabolomics identifies metabolite alterations that precede subclinical mastitis in the blood of transition dairy vows. J. Proteome Res. 16:433-446.

Dervishi, E., G. Zhang, R. Mandal, D. S. Wishart, and B. N. Ametaj. 2017b. 043 Metabolomics uncovers serum biomarkers that can predict the risk of retained placenta in transition dairy cows. J. Anim. Sci. 95(Supplement4):21-22.

Drackley, J. K. 1999. Biology of dairy cows during the transition period: The final frontier? J. Dairy Sci. 82:2259-2273.

Esposito, G., P. C. Irons, E. C. Webb, and A. Chapwanya. 2014. Interactions between negative energy balance, metabolic diseases, uterine health and immune response in transition dairy cows. Anim. Reprod. Sci. 144:60-71.

Gandra, J. R., J. E. D. Freitas Junior., M. Maturna Filho, R. V. Barletta, L. C. Verdurico, and F. P. Rennó. 2014. Soybean oil and calcium salts of fatty acids as fat sources for Holstein dairy cows in transition period. Rev. Bras. Saúde Prod. Anim. 15:83-93.

Giallongo, F., M. T. Harper, J. Oh, J. C. Lopes, H. Lapierre, R. A. Patton, C. Parys, I. Shinzato, and A. N. Hristov. 2016. Effects of rumen-protected methionine, lysine, and histidine on lactation performance of dairy cows. J. Dairy Sci. 99:4437-4452.

Grummer, R. R., D. G. Mashek, and A. Hayirli. 2004. Dry matter intake and energy balance in the transition period. Vet. Clin. North Am. Food Anim. Pract. 20:447-470.

Gulliver, C. E., M. A. Friend, B. J. King, and E. H. Clayton. 2012. The role of omega-3 polyunsaturated fatty acids in reproduction of sheep and cattle. Anim. Reprod. Sci. 131:9-22.

Imhasly, S., H. Naegeli, S. Baumann, M. V. Bergen, A. Luch, H. Jungnickel, S. Potratz, and C. Gerspach. 2014. Metabolomic biomarkers correlating with hepatic lipidosis in dairy cows. BMC Vet. Res. $10: 122$.

Jia, H., X. Shen, Y. Guan, M. Xu, J. Tu, M. Mo, L. Xie, J. Yuan, Z. Zhang, S. Cai, J. Zhu, and Z. Zhu. 2018. Predicting the pathological response to neoadjuvant chemoradiation using untargeted metabolomics in locally advanced rectal cancer. Radiother. Oncol. 128:548-556.

Jiao, N., Z. Wu, Y. Ji, B. Wang, Z. Dai, and G. Wu. 2015. L-Glutamate enhances barrier and antioxidative functions in intestinal porcine epithelial cells. J. Nutr. 145:2258-2264.

Kenéz, Á., S. Dänicke, U. Rolle-Kampczyk, M. von Bergen, and K. Huber. 2016. A metabolomics approach to characterize phenotypes of metabolic transition from late pregnancy to early lactation in dairy cows. Metabolomics 12:165-176.

Khatti, A., S. Mehrotra, P. K. Patel, G. Singh, V. P. Maurya, A. S. Mahla, R. K. Chaudhari, G. K. Das, M. Singh, M. Sarkar, H. Kumar, and N. Krishnaswamy. 2017. Supplementation of vitamin E, selenium and increased energy allowance mitigates the transition stress and improves postpartum reproductive performance in the crossbred cow. Theriogenology 104(Supplement C):142-148.

Lee, C., F. Giallongo, A. N. Hristov, H. Lapierre, T. W. Cassidy, K. S. Heyler, G. A. Varga, and C. Parys. 2015. Effect of dietary protein level and rumen-protected amino acid supplementation on amino acid utilization for milk protein in lactating dairy cows. J. Dairy Sci. 98:1885-1902.

Leng, X., J. J. Kinnun, A. T. Cavazos, S. W. Canner, S. R. Shaikh, S. E. Feller, and S. R. Wassall. 2018. All n-3 PUFA are not the same: MD simulations reveal differences in membrane organization for EPA, DHA and DPA. Biochim. Biophys. Acta Biomembr. 1860:1125-1134.

Li, S., Q. Wang, X. Lin, X. Jin, L. Liu, C. Wang, Q. Chen, J. Liu, and H. Liu. 2017. The use of "Omics" in lactation research in dairy cows. Int. J. Mol. Sci. 18:983-1000.
Liang, M., Z. Wang, H. Li, L. Cai, J. Pan, H. He, Q. Wu, Y. Tang, J. Ma, and L. Yang. 2018. l-Arginine induces antioxidant response to prevent oxidative stress via stimulation of glutathione synthesis and activation of Nrf2 pathway. Food Chem. Toxicol. 115:315-328.

Liu, H., F. Tayyari, C. Khoo, and L. Gu. 2015. A 1 H NMR-based approach to investigate metabolomic differences in the plasma and urine of young women after cranberry juice or apple juice consumption. J. Funct. Foods. 14(Jan16 2):76-86.

Marion-Letellier, R., G. Savoye, and S. Ghosh. 2015. Polyunsaturated fatty acids and inflammation. IUBMB Life 67:659-667.

Mauvais-Jarvis, F., D. J. Clegg, and A. L. Hevener. 2013. The role of estrogens in control of energy balance and glucose homeostasis. Endocr. Rev. 34:309-338.

Mavangira, V., J. C. Gandy, C. Zhang, V. E. Ryman, A. Daniel Jones, and L. M. Sordillo. 2015. Polyunsaturated fatty acids influence differential biosynthesis of oxylipids and other lipid mediators during bovine coliform mastitis. J. Dairy Sci. 98:6202-6215.

Mavangira, V., and L. M. Sordillo. 2018. Role of lipid mediators in the regulation of oxidative stress and inflammatory responses in dairy cattle. Res. Vet. Sci. 116:4-14.

Nicholson, J. K., J. C. Lindon, and E. Holmes. 1999. 'Metabonomics': Understanding the metabolic responses of living systems to pathophysiological stimuli via multivariate statistical analysis of biological NMR spectroscopic data. Xenobiotica 29:1181-1189.

Overton, T. R., J. A. A. McArt, and D. V. Nydam. 2017. A 100-Year Review: Metabolic health indicators and management of dairy cattle. J. Dairy Sci. 100:10398-10417.

Park, S. Y., J. Ryu, and W. Lee. 2005. O-GlcNAc modification on IRS-1 and Akt2 by PUGNAc inhibits their phosphorylation and induces insulin resistance in rat primary adipocytes. Exp. Mol. Med. 37:220-229.

Patti, G. J., O. Yanes, and G. Siuzdak. 2012. Innovation: Metabolomics: The apogee of the omics trilogy. Nat. Rev. Mol. Cell Biol. 13:263-269.

Raphael, W., L. Halbert, G. A. Contreras, and L. M. Sordillo. 2014. Association between polyunsaturated fatty acid-derived oxylipid biosynthesis and leukocyte inflammatory marker expression in periparturient dairy cows. J. Dairy Sci. 97:3615-3625.

Roach, P. J., A. A. Depaoliroach, T. D. Hurley, and V. S. Tagliabracci. 2012. Glycogen and its metabolism: Some new developments and old themes. Biochem. J. 441:763-787.

Robinson, P. H., N. Swanepoel, and E. Evans. 2010. Effects of feeding a ruminally protected lysine product, with or without isoleucine, valine and histidine, to lactating dairy cows on their productive performance and plasma amino acid profiles. Anim. Feed Sci. Technol. 161:75-84.

Ryman, V. E., N. Packiriswamy, and L. M. Sordillo. 2016. Apoptosis of endothelial cells by 13-HPODE contributes to impairment of endothelial barrier integrity. Mediators Inflamm. 2016:9867138.

Ryman, V. E., G. M. Pighetti, J. D. Lippolis, J. C. Gandy, C. M. Applegate, and L. M. Sordillo. 2015. Quantification of bovine oxylipids during intramammary Streptococcus uberis infection. Prostaglandins Other Lipid Mediat. 121(Pt B):207-217.

Salin, S., A. Vanhatalo, K. Elo, J. Taponen, R. C. Boston, and T. Kokkonen. 2017. Effects of dietary energy allowance and decline in dry matter intake during the dry period on responses to glucose and insulin in transition dairy cows. J. Dairy Sci. 100:5266-5280.

Schäfers, S., D. S. Von, U. Meyer, C. Drong, J. Frahm, A. Tröscher, W. Pelletier, H. Sauerwein, and S. Dänicke. 2018. Influence of conjugated linoleic acids and vitamin $\mathrm{E}$ on biochemical, hematological, and immunological variables of dairy cows during the transition period. J. Dairy Sci. 101:1585-1600.

Sharma, N., N. K. Singh, O. P. Singh, V. Pandey, and P. K. Verma. 2011. Oxidative stress and antioxidant status during transition period in dairy cows. Asian-Australas. J. Anim. Sci. 24:479-484.

Sies, H., C. Berndt, and D. P. Jones. 2017. Oxidative stress. Annu. Rev. Biochem. 86:715-748.

Sordillo, L. M., and V. Mavangira. 2014. The nexus between nutrient metabolism, oxidative stress and inflammation in transition cows. Anim. Prod. Sci. 54:1204-1214. 
Stein, S. E., and D. R. Scott. 1994. Optimization and testing of mass spectral library search algorithms for compound identification. J. Am. Soc. Mass Spectrom. 5:859-866.

Sugiyama, K., H. Ebinuma, N. Nakamoto, N. Sakasegawa, Y. Murakami, P. S. Chu, S. Usui, Y. Ishibashi, Y. Wakayama, N. Taniki, H. Murata, Y. Saito, M. Fukasawa, K. Saito, Y. Yamagishi, T. Wakita, H. Takaku, T. Hibi, H. Saito, and T. Kanai. 2014. Prominent steatosis with hypermetabolism of the cell line permissive for years of infection with hepatitis C virus. PLoS One 9:e94460.

Sun, L. W., H. Y. Zhang, L. Wu, S. Shu, C. Xia, C. Xu, and J. S. Zheng. 2014. (1)H-Nuclear magnetic resonance-based plasma metabolic profiling of dairy cows with clinical and subclinical ketosis. J. Dairy Sci. 97:1552-1562.

Trevisi, E., M. Amadori, S. Cogrossi, E. Razzuoli, and G. Bertoni. 2012. Metabolic stress and inflammatory response in high-yielding, periparturient dairy cows. Res. Vet. Sci. 93:695-704.

Urasaki, Y., G. Pizzorno, and T. T. Le. 2014. Uridine affects liver protein glycosylation, insulin signaling, and heme biosynthesis. PLoS One 9:e99728.

Wankhade, P. R., A. Manimaran, A. Kumaresan, S. Jeyakumar, K. P. Ramesha, V. Sejian, D. Rajendran, and M. R. Varghese. 2017. Metabolic and immunological changes in transition dairy cows: A review. Vet. World 10:1367-1377.

Westerhuis, J. A., H. C. Hoefsloot, S. Smit, D. J. Vis, A. K. Smilde, E. J. van Velzen, J. P. van Duijnhoven, and F. A. van Dorsten. 2008. Assessment of PLSDA cross validation. Metabolomics 4:81-89.
Yoshimi, N., T. Futamura, K. Kakumoto, A. M. Salehi, C. M. Sellgren, J. Holmen-Larsson, J. Jakobsson, E. Palsson, M. Landen, and K. Hashimoto. 2016. Blood metabolomics analysis identifies abnormalities in the citric acid cycle, urea cycle, and amino acid metabolism in bipolar disorder. BBA Clin. 5:151-158.

Zhang, G., Q. Deng, R. Mandal, D. S. Wishart, and B. N. Ametaj. 2017a. DI/LC-MS/MS-based metabolic profiling for identification of early predictive serum biomarkers of metritis in transition dairy cows. J. Agric. Food Chem. 65:8510-8521.

Zhang, G., E. Dervishi, S. M. Dunn, R. Mandal, P. Liu, B. Han, D. S. Wishart, and B. N. Ametaj. 2017b. Metabotyping reveals distinct metabolic alterations in ketotic cows and identifies early predictive serum biomarkers for the risk of disease. Metabolomics 13:43-58.

Zhang, H., W. Ling, C. Xu, X. Cheng, L. Sun, and S. Shi. 2013. Plasma metabolomic profiling of dairy cows affected with ketosis using gas chromatography/mass spectrometry. BMC Vet. Res. 9:186-199.

Zhao, F. F., T. Y. Wu, H. R. Wang, L. Y. Ding, G. Ahmed, H. W. Li, W. Tian, and Y. Z. Shen. 2018. Jugular arginine infusion relieves lipopolysaccharide-triggered inflammatory stress and improves immunity status of lactating dairy cows. J. Dairy Sci. 101:5961-5970.

Zhuge, Y., Y. Yuan, R. van Breemen, M. Degrand, O. Holian, M. Yoder, and H. Lum. 2014. Stimulated bronchial epithelial cells release bioactive lysophosphatidylcholine 16:0, 18:0, and 18:1. Allergy Asthma Immunol. Res. 6:66-74. 